\title{
Determination of gain of double-ridged guide horn antenna by considering phase center
}

\author{
Katsushige Harima ${ }^{a}$ \\ National Institute of Information and Communications Technology, \\ 4-2-1, Nukui-Kitamachi, Koganei, Tokyo 184-8795, Japan \\ a) harima@nict.go.jp
}

Abstract: The far-field gain of a double-ridged guide horn antenna is determined by the three-antenna method by considering the phase center. The location of the phase center is calculated by the finite integration method. The experimental and theoretical results show the efficacy of using the phase center for antenna calibration.

Keywords: double-ridged guide horn antenna, phase center, gain calibration, three-antenna method, finite integration method

Classification: Electromagnetic compatibility (EMC)

\section{References}

[1] T. S. Chu and R. A. Semplak, "Gain of electro-magnetic horns," Bell Syst. Tech. J., vol. 44, no. 3, pp. 527-537, March 1965.

[2] K. Harima, M. Sakasai, and K. Fujii, "Determination of gain for pyramidal-horn antenna on basis of phase center location," Proc. 2008 IEEE Int. Symp. Electromagn. Compat., Detroit, USA, pp. 1-5, Aug. 2008.

[3] E. I. Muehldorf, "The phase center of horn antennas," IEEE Trans. Antennas Propag., vol. AP-18, no. 6, pp. 753-160, Nov. 1970.

[4] C. Bruns, P. Leuchtmann, and R. Vahldieck, "Analysis and simulation of a $1-18 \mathrm{GHz}$ broadband double-ridged horn antenna," IEEE Trans. Electromagn. Compat., vol. 45, no. 1, pp. 55-60, Feb. 2003.

[5] D. Baumann, C. Fumeaux, P. Leuchtmann, and R. Vahldieck, "Finitevolume time-domain (FVTD) modeling of a broadband double-ridged horn antenna," Int. J. Numer. Model., pp. 285-298, 2004.

[6] M. B. Perez, H. J. Aguilar, and I. G. Ruiz, "Design and simulation of a 1 to $14 \mathrm{GHz}$ broadband electromagnetic compatibility DRGH antenna," Proc. 2nd Int. Conf. Electrical and Electron. Engineering, Mexico City, Mexico, pp. 118-121, Sept. 2005.

[7] M. A. Azimi, F. Arazm, and R. F. Dana, "Design and optimization of a high-frequency EMC wideband horn antenna," IRE Trans. Microw. Antenna Propag., vol. 1, no. 3, pp. 580-585, June 2007.

[8] L. Wei and X. XiaoLi, "Design and simulation of TEM double ridge guide horn antenna," Proc. the 8th Int. Conf. Electronic Measurement and Instructions, Xi'an, China, pp. 703-706, 2007.

[9] K. Harima, "Calibration of double-ridged guide horn antenna using phase center," Proc. 2009 IEEE Int. Symp. Electromagn. Compat., Austin, 
USA, pp. 287-291, Aug. 2009.

[10] MW-Studio, ver. 2008, CST, Darmstadt, Germany.

[11] J. P. Berebger, "A perfectly matched layer for the absorption of electromagnetic waves," J. Computational Physics, vol. 114, pp. 185-200, 1994.

[12] A. R. Panicali and M. M. Nakamura, "On the amplitude center of radiating apertures," IEEE Trans. Antennas Propag., vol. AP-33, no. 3, pp. 330-335, March 1985.

\section{Introduction}

The three-antenna method is often used to determine the far-field gain of aperture antennas. Usually, the distance between the apertures of two antennas is used for gain measurement. The gain of horn antennas varies depending on the separation distance, even if it satisfies the far-field criterion required for gain measurement. Chu [1] derived a correction for this gain variation as a function of the antenna dimensions and the distance between the apertures of the two horns. The phase center of the horn antenna is considered an equivalent point source under a far field condition. Using the distance between the phase centers is suitable for dealing with the antenna separation distance in the three-antenna method [2]. Muehldorf [3] determined the phase centers of horns by a calculation based on a vector approach.

Double-ridged guide horn antennas are widely used as wideband antennas in electromagnetic compatibility (EMC) testing. It is difficult to determine the gain variation and the phase center in the case of the ridged horns by the theoretical approach $[1,3]$ used in the case of horn antennas; this is because in the case of ridged horn antennas, the antenna structure is complex. The directivity and gain characteristics of ridged horn antennas have been determined by several numerical simulations, e.g., the moment method (MoM) [4], the finite-volume time-domain (FVTD) method [5], the finite-difference timedomain (FDTD) method [6], the finite element method (FEM) [7], and the finite integration method (FIM) [7,8]. Recently, the use of the phase center of a ridged horn antenna to determine the gain has been investigated; however, the effect of measurement distance on the accuracy of the value obtained is not clear [9].

In this paper, we discuss the effect of the measurement distance on the gain determination for a double-ridged guide horn antenna by considering the locations of the aperture and phase center. Experimental and numerical results indicate a significant relationship between the phase center and the effect of antenna separation on gain.

\section{Three-antenna method}

Two antennas are arranged under free space and far field conditions, as shown in Fig. 1. Then, the relationship between the insertion loss $\left(A_{t r}\right)$, which is defined as the ratio of the power received $\left(P_{r}\right)$ by the receiving antenna to the 


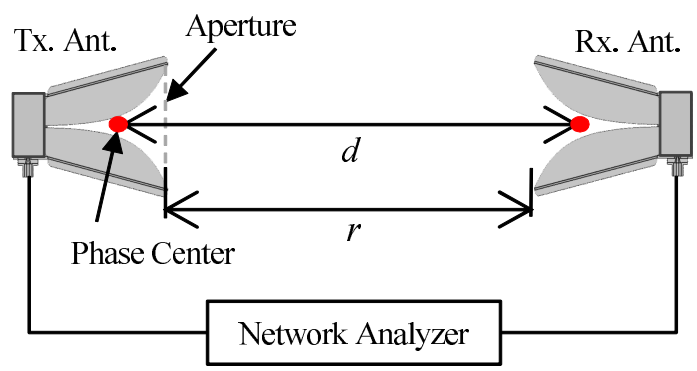

Fig. 1. Setup for the three-antenna method.

input power $\left(P_{t}\right)$ of the transmitting antenna, and the gain can be expressed by using the Friis transmission formula:

$$
G_{t} G_{r}=P_{r} / P_{t}\left(\frac{4 \pi r}{\lambda}\right)^{2}=A_{t r}\left(\frac{4 \pi r}{\lambda}\right)^{2}
$$

Here, $G_{t}$ is the gain of the transmitting antenna; $G_{r}$, the gain of the receiving antenna; $r$, the distance between the two antennas; and $\lambda$, the wavelength.

The gain of each antenna can be determined by solving three equations (1) obtained by using all the combinations of the three antennas. For example, the gain of one of the three antennas is expressed as follows.

$$
G_{1}=\frac{4 \pi r}{\lambda} \sqrt{\frac{A_{12} A_{13}}{A_{23}}} .
$$

The distance between the apertures is often considered the antenna separation distance. However, during gain measurement, it is suitable to consider the distance between the phase centers as the antenna separation. This is because the phase center, which is defined as the center of curvature of the equiphase front, is treated an equivalent point source of far-field radiation.

\section{Phase center of ridged horn}

The phase center of the conventional double-ridged guide horn was determined by using a full-wave electromagnetic solver, CST MW-Studio [10], which is based on FIM. The location of the phase center was computed from the equiphase pattern, which was calculated by adjusting the origin of the near- to far-field transformation along the bore sight.

The antenna used for this evaluation was a commercial ridged horn with an operating frequency range $1-18 \mathrm{GHz}$. The calculation model of the ridged horn is shown in Fig. 2 (a). The analytical region consisted of 23841930 $(=210 \times 331 \times 343)$ nonuniform cells with a magnetic wall, and the maximum cell size was $\lambda / 10$. The boundaries of the analytical region were assumed to be absorbing boundaries. The perfectly matched layer (PML) with four layers considered as the absorbing boundary condition [11]. The calculation model was assumed to consist of actual materials used in an antenna, i.e., mainly aluminum and fiberglass substrate, and was excited by a coaxial feed.

Figure 2 (b) shows the location of the phase center of the ridged horn; the locations of the $\mathrm{H}$ and $\mathrm{E}$ planes are determined by considering an angular 


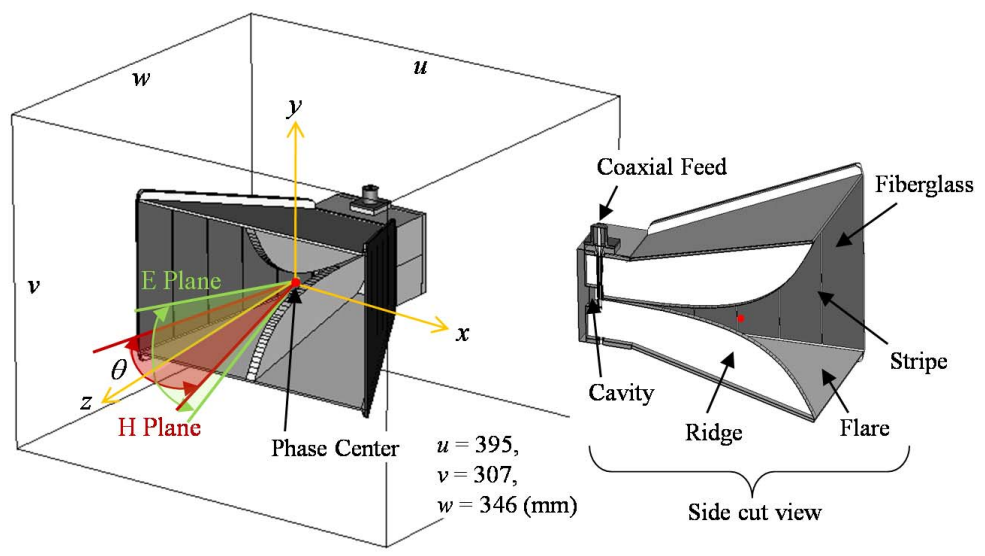

(a)

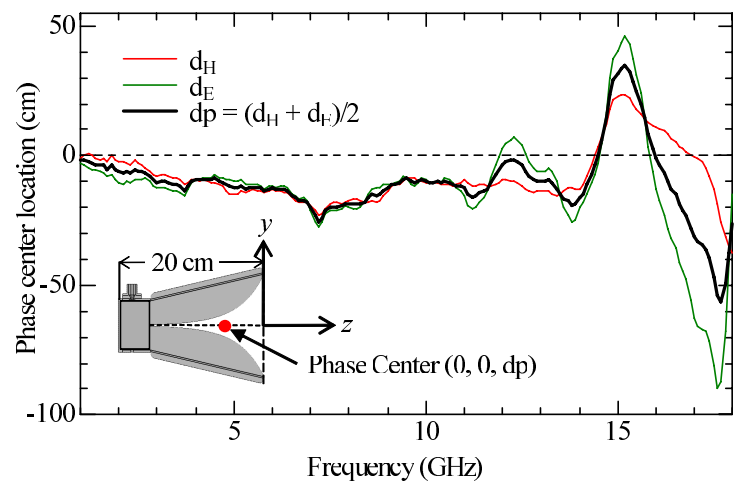

(b)

Fig. 2. (a) Calculation model of a commercial ridged horn. (b) The locations of phase center $\left(d_{E}\right.$ and $\left.d_{H}\right)$ relative to the aperture for $\mathrm{E}$ and $\mathrm{H}$ planes.

region of $4^{\circ}(=\theta)$. The phase center is sensitive to changes in the frequency, and at frequencies above $12 \mathrm{GHz}$, it is located outside the antenna. For gain determination, the mean of the locations of the phase centers of the $\mathrm{H}$ and E planes is considered [12].

The changes $(\Delta G)$ in the gains determined by considering the distance between the phase centers $\left(G_{d}\right)$ and that between the apertures $\left(G_{r}\right)$ in the three-antenna method depends only on the ratio of these distances; this ratio is derived from (2) as follows:

$$
\Delta G=G_{r} / G_{d}=r / d .
$$

\section{Experimental results}

The transmitting and receiving antennas were separated by a distance $r$ and were placed in a full anechoic chamber so that they faced each other. The antennas were connected to a network analyzer (Fig. 1). Three ridged horns, which were similar to those considered in the calculations, were used in the three-antenna method. We measured the antenna insertion (S21 parameter) and impedance mismatch (S11 parameter) losses of the three pairs of antennas by using the network analyzer and determined the gain from (2). 
Figures $3(\mathrm{a})$ and (b) show the gains $\left(G_{d}\right.$ and $\left.G_{r}\right)$ measured by considering the locations of the aperture and phase center for antenna separations $(r)$ of 1 , 3 , and $15 \mathrm{~m}$; the measured gains were compared with far-field gains calculated

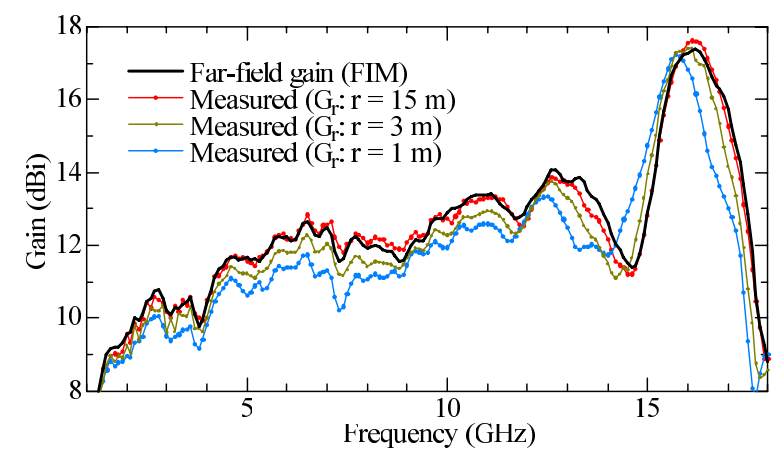

(a)

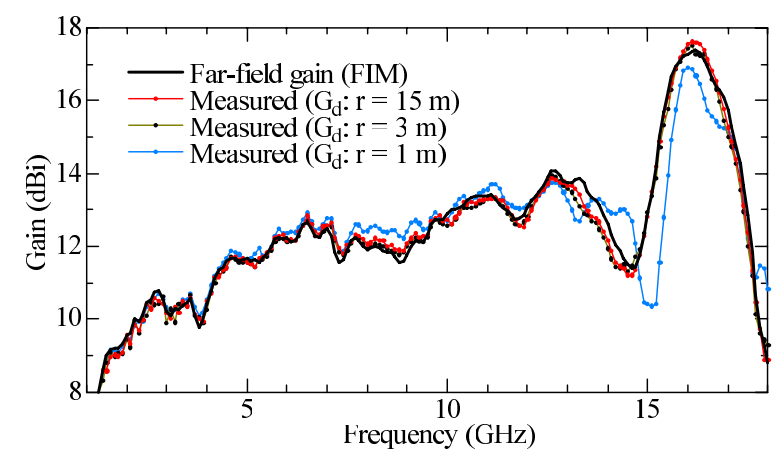

(b)

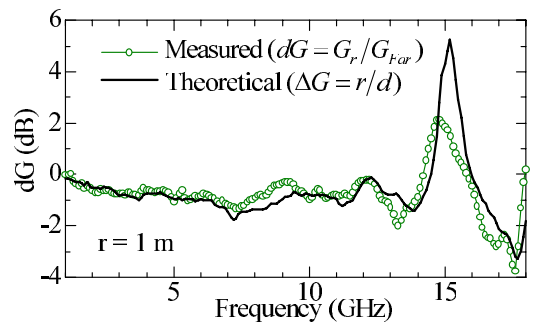

(c)

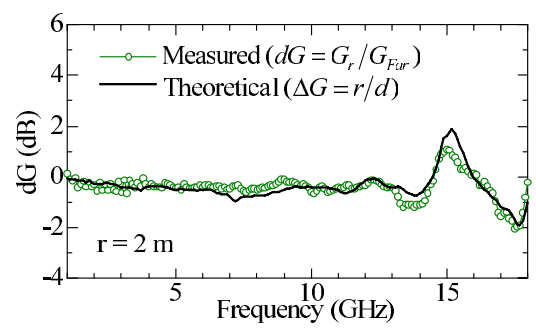

(e)

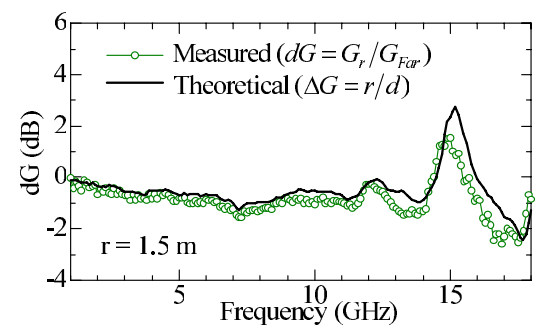

(d)

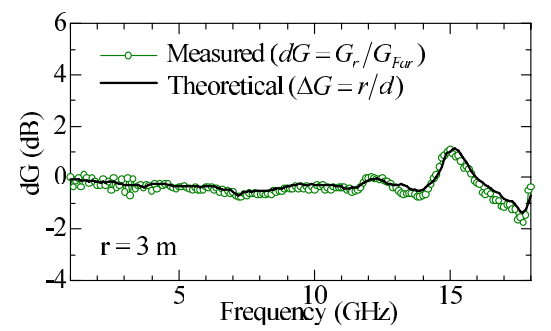

(f)

Fig. 3. Gains of a ridged horn measured by considering the locations of (a) the aperture and (b) phase center. The ratio $(d G)$ of the gain $\left(G_{r}\right)$ determined by considering the aperture to the farfield gain $\left(G_{\text {Far }}\right)$ for aperture separations of (c) $r=1 \mathrm{~m}$, (d) $r=1.5 \mathrm{~m}$, (e) $r=2 \mathrm{~m}$, and (f) $r=3 \mathrm{~m}$. 
by the FIM (shown in the same figure). The gain determined by using the location of the aperture, i.e., by using the conventional three-antenna method, varied according to the measurement distance. Although this gain variation is negligible if the distance between the antennas is sufficiently large, (e.g., $15 \mathrm{~m}$ ), the maximum variation in the gain at an aperture separation of $3 \mathrm{~m}$ is approximately $1 \mathrm{~dB}$, as shown in Fig. 3 (a). On the other hand, the gain determined by considering the location of the phase center at the relatively small separation of $3 \mathrm{~m}$ was in good agreement with the far-field gain, as shown Fig. 3 (b). However, the gain measured at a short distance (e.g., $1 \mathrm{~m}$ ) was sensitive to the near-field effect.

The changes $(d G)$ in the gain $\left(G_{r}\right)$ determined by considering the distance between the apertures and the measured far-field gain $\left(G_{\text {Far }}\right)$ for antenna separations of 1 to $3 \mathrm{~m}$ are shown in Figs. 3 (c)-(f). The gain determined by considering the location of the phase center at the distance of $15 \mathrm{~m}$ was used as the far-field gain. The ratio $(\Delta G)$ of the distance between the apertures and the distance between the phase centers, expressed in (3), is plotted in the same figures. The difference between the gain variation $(d G)$ and the distance ratio $(\Delta G)$ decreased as the measurement distance increased, and both were in good agreement at the measurement distance of $3 \mathrm{~m}$. Hence, when the gain is determined on the basis of the location of the aperture, the changes in gain due to the measurement distance is equal to the ratio of the phase center-to-phase center separation to the aperture-to-aperture separations, i.e.,

$$
d G=G_{r} / G_{F a r} \simeq \Delta G=r / d .
$$

These results indicate that in place of using the conventional threeantenna method, which considers the location of the aperture and requires considerable antenna separation to determine the far-field gain, the ridged guide horn antenna can be calibrated at small aperture separations (e.g., 3 $\mathrm{m})$ by considering the location of the phase center.

\section{Conclusion}

We described the determination of the gain of a double-ridged guide horn antenna by the three-antenna method in which the phase center is considered. The phase center was computed by the finite integration method. Experimental and numerical results indicate the following: (1) the gain determined by considering the phase center is in good agreement with the far-field gain, even at relatively small separation distance of $3 \mathrm{~m},(2)$ the gain determined by considering the aperture varies depending on the measurement distance, and (3) this gain variation is equal to the ratio of distance between the apertures and that between the phase centers. 